\title{
The Effect of Paclitaxel Drug-coating on the Balloon Performances
}

\author{
Shi Xiao Man ${ }^{1,2, a}$, Lu Chun Lan ${ }^{1, b}$ and Li Xin Yue $e^{1,2, c^{*}}$ \\ ${ }^{1}$ College of Environmental and Chemical Engineering, Dalian University, Dalian 116622, China \\ ${ }^{2}$ Yinyi(Liaoning) Biotech. Co., Ltd, Dalian 116100, China \\ asxmhome@126.com, 'Iclsophia@163.com, chomebox11@126.com
}

Keywords: Paclitaxel, Balloon, Drug-coated

Abstract. The nylon-12 as raw material of balloon catheter and paclitaxel as coating drug, the paclitaxel drug-coating balloon was made by directing coating method. This paper investigated the effect of paclitaxel drug-coating on the balloon physical performances by Hydraulic Pressure Tester, Laser Diameter Gauge, and further investigated changes of paclitaxel and balloon surface by High Performance Liquid Chromatography (HPLC) and Fourier Transform Infrared Spectrometer (FTIR) experiments. Analyzed the test results, it could be found that the physical performances and structure of the balloon surface and paclitaxel properties haven't been changed after the balloon was coated, the coated balloon still has excellent performances.

\section{Introduction}

The research of paclitaxel drug-coating balloon began in $1987^{[1]}$, which was a novel therapeutic drug delivery technology and has been developed on the basis of interventional treatment including balloon dilation and balloon angioplasty. Coating the anti-intimal hyperplasia drug on the surface of balloon, this drug can be transferred to the local vascular wall by tearing vessel film and pressuring rapidly release, when the drug-coated balloon reached to the diseased vessel and distracted, expanded and contacted with the vessel wall ${ }^{[2,3]}$. Drugs have played the role of anti-intimal hyperplasia, thereby preventing vascular restenosis ${ }^{[4,5]}$.

Now paclitaxel is regarded as the most effective drug for curing vascular restenosis. Paclitaxel is a natural extract of yew playing a significant role in treating the anti-cell hyperplasia. It can hinder mitosis of the smooth muscle cell by affecting the microvascular function, thus inhibiting neointimal hyperplasia, in order to achieve different degrees inhibition for restenosis ${ }^{[6-8]}$.

Paclitaxel drug-coated balloon (Fig. 1) can effectively cure and prevent vascular restenosis, but there are few studies of its underlying theory. This article focuses on studying and discussing the performances of the paclitaxel drug-coated balloon.

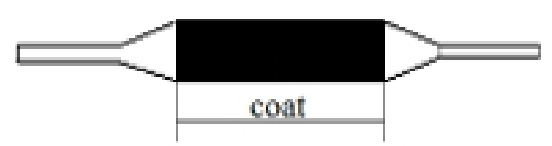

Fig. 1 Paclitaxel drug-coated balloon

\section{Experimental Part}

Materials and Equipments. Balloon raw material: Nylon-12; Paclitaxel: Purity $\square$ 98\%; Hydraulic Pressure Tester: PT-500; Laser Diameter Gauge: LDSMY-10B; FTIR: Nicolet 550; HPLC: Hitachi L-2000.

Preparation of the Sample. Three specification paclitaxel drug-coated balloons were prepared 
by directing coating method ${ }^{[9]}$, which are 2010, 2515 and 3020 (the first two digits represent the balloon diameter $/ 0.1 \mathrm{~mm}$, the end two digits represent the balloon length $/ \mathrm{mm}$ ).

\section{Performance Test}

Balloon size.The test of balloon size refers to measure the diameter and length of the balloon. Inflating the balloon to the nominal pressure (10atm), balloon diameter and length were measured by Laser Diameter Gauge and Digital Vernier Caliper, respectively.

Balloon crossing profile. Balloon crossing profile is an important factor for the balloon catheter clinical application, i.e. the outer diameter of the folded balloon. The smaller balloon crossing profile is, the more smoothly passing the narrow lesion is. Balloon crossing profile was obtained by Digital Vernier Caliper under exhausted negative pressure condition.

Balloon burst pressure. Balloon burst pressure refers to the inflated pressure when a balloon burst. To measure the balloon burst pressure, Hydraulic Pressure Tester was carried out. Balloon was dipping in $37 \square$ water bath for least $2 \mathrm{~min}$, pressurizing at $1.0 \mathrm{~atm} / \mathrm{s}$ speeds and staying $10 \mathrm{~s}$, continuing inflating balloon until the balloon burst and recording the maximum pressure.

Balloon Compliance. Compliance is the ratio of the difference between the burst pressure and the nominal pressure of the balloon and the diameter under the nominal pressure, used to characterize the degree of the diameter change of balloon during filling pressure to the balloon ${ }^{[10]}$, showed by equation $(1)^{[11]}$.

$$
\text { Compliance }=\frac{\Phi D_{\text {Explosion }}-\Phi D_{\text {Normal }}}{\Phi D_{\text {Normal }}}
$$

$\Phi D_{\text {Explosion: }}$ the diameter under the rated burst pressure of the balloon, $\mathrm{mm}$.

$\Phi D_{\text {Normal }}$ : the diameter under rated the normal pressure of the balloon, $\mathrm{mm}$.

Qualitative Analysis of Paclitaxel. To characterize paclitaxel, the retention time of the paclitaxel coated and paclitaxel standard were measured by HPLC. If the retention time of the paclitaxel coated is $90 \sim 110 \%$ of the paclitaxel standard retention time, it can be characterized as paclitaxel $^{[12]}$. Setting paclitaxel coated balloon into chromatography ethanol, and taking the ethanol solution as tested sample.

Test conditions: column $\mathrm{C}_{18}(4.6 \times 250 \mathrm{~mm}, 5 \mu \mathrm{m})$, detection wavelength $227 \mathrm{~nm}$, injection volume $1 \mu \mathrm{L}$, flowing rate of $1.2 \mathrm{~mL} / \mathrm{min}$, column temperature $45 \square$, the mobile phase of acetonitrile-water ratio 60:40.

FTIR Spectrometer. Surface functional groups were measured by FTIR Spectrometer from Nicolet 550. One hundred and twenty-eight scans were collected with a resolution of $4 \mathrm{~cm}^{-1}$. Paclitaxel coated balloon completely was immersed in ethanol, dissolved the drug of balloon surface, dried and set aside. Cutting the sample and uncoated balloon into strips and fixing on the sample holder for infrared experiments.

\section{Results and Discussion}

Three specification balloons which are 2010, 2515 and 3020 were tested in experiments, wherein each test each specification is taken five samples. Then the average value was obtained.

Effect of paclitaxel drug-coating on the balloon diameter. After coating paclitaxel, the 
balloon diameter was slightly increased about $0.04 \mathrm{~mm}$ as shown in Table 1 . Increased diameter is the thickness of paclitaxel coated. In addition, the relative standard deviations (RSD) of paclitaxel drug-coated balloon diameter are still less than $1 \%$, it indicated that the coating on balloon surface is homogeneous.

Table 1 Diameter of uncoated balloon and coated balloon

\begin{tabular}{ccccc}
\hline Balloon & \multicolumn{2}{c}{ Uncoated balloon } & \multicolumn{2}{c}{ Coated balloon } \\
\cline { 2 - 5 } specification & Ave.[mm] & RSD[\%] & Ave.[mm] & RSD[\%] \\
\hline 2010 & 2.01 & 0.56 & 2.05 & 0.55 \\
2515 & 2.50 & 0.48 & 2.54 & 0.49 \\
3020 & 3.01 & 0.43 & 3.05 & 0.42 \\
\hline
\end{tabular}

Effect of paclitaxel drug-coating on the balloon length. It can be seen in the Table 2 the average value and relative standard deviation of the coated balloon length haven't been changed. So it can be concluded that paclitaxel coating has no influence on balloon length.

Table 2 Length of uncoated balloon and coated balloon

\begin{tabular}{ccccc}
\hline Balloon & \multicolumn{2}{c}{ Uncoated balloon } & \multicolumn{2}{c}{ Coated balloon } \\
\cline { 2 - 5 } specification & Ave.[mm] & RSD[\%] & Ave.[mm] & RSD[\%] \\
\hline 2010 & 10.6 & 0.36 & 10.6 & 0.36 \\
2515 & 15.3 & 0.40 & 15.2 & 0.41 \\
3020 & 20.6 & 0.26 & 20.6 & 0.26 \\
\hline
\end{tabular}

Effect of paclitaxel drug-coating on the balloon crossing profile. The outer diameter of folded balloon were recorded in Table 3. There are hardly change in the average value and relative standard deviation after balloon was coated. It proved that coated balloon still can cross the narrow vascular lesion in clinical.

Table 3 Crossing profile of uncoated balloon and coated balloon

\begin{tabular}{ccccc}
\hline \multirow{2}{*}{$\begin{array}{c}\text { Balloon } \\
\text { specification }\end{array}$} & \multicolumn{2}{c}{ Uncoated balloon } & \multicolumn{2}{c}{ Coated balloon } \\
\cline { 2 - 5 } & Ave.[mm] & RSD[\%] & Ave.[mm] & RSD[\%] \\
\hline 2010 & 0.68 & 0.28 & 0.70 & 0.29 \\
2515 & 0.81 & 0.44 & 0.83 & 0.45 \\
3020 & 0.94 & 0.36 & 0.96 & 0.34 \\
\hline
\end{tabular}

Effect of paclitaxel drug-coating on the balloon burst pressure. Table 4 shows the uncoated balloon and coated balloon average burst pressure are both in the range of 25 26atm. The coated balloon burst pressure hasn't been changed and the relative standard deviation is still less than 4\%, So these demonstrated that paclitaxel coated balloon still has a certain stability.

Table 4 Burst pressure of uncoated balloon and coated balloon

\begin{tabular}{ccccc}
\hline \multirow{2}{*}{$\begin{array}{c}\text { Balloon } \\
\text { specification }\end{array}$} & \multicolumn{2}{c}{ Uncoated balloon } & \multicolumn{2}{c}{ Coated balloon } \\
\cline { 2 - 5 } & Ave.[atm] & RSD[\%] & Ave.[atm] & RSD[\%] \\
\hline 2010 & 25 & 2.28 & 25 & 2.28 \\
2515 & 25 & 3.24 & 25 & 3.24 \\
3020 & 26 & 2.71 & 26 & 2.71 \\
\hline
\end{tabular}

Effect of paclitaxel drug-coating on the balloon compliance. Balloon compliance was obtained by the compliance formula based on the rated nominal pressure and rated burst pressure, as can been seen in Table 5. The coated balloon average compliance is $10.0 \sim 12.0 \%$ and relative 
standard deviation is in close proximity to uncoated balloon's. So the paclitaxel coating has no effect on the balloon compliance.

Table 5 Compliance of uncoated balloon and coated balloon

\begin{tabular}{ccccc}
\hline \multirow{2}{*}{ Balloon specification } & \multicolumn{2}{c}{ Uncoated balloon } & \multicolumn{2}{c}{ Coated balloon } \\
\cline { 2 - 5 } & Ave.[\%] & RSD[\%] & Ave.[\%] & RSD[\%] \\
\hline 2010 & 10.8 & 1.73 & 10.8 & 1.73 \\
2515 & 11.4 & 1.62 & 11.3 & 1.64 \\
3020 & 10.1 & 2.05 & 10.1 & 2.04 \\
\hline
\end{tabular}

Qualitative analysis of paclitaxel. HPLC clearly shows that the retention times of paclitaxel standard and paclitaxel coated in Fig. 2, which are 9.68 and 9.70min, respectively. Although two retention time are different, the paclitaxel coated retention time is $100.2 \%$ of paclitaxel standard, and the change still stays the range of $90 \sim 110 \%$. So it can be regarded as paclitaxel. The reason of retention time change may be dissolving process, coating process and balloon material lead certain extent impacting for paclitaxel coated.
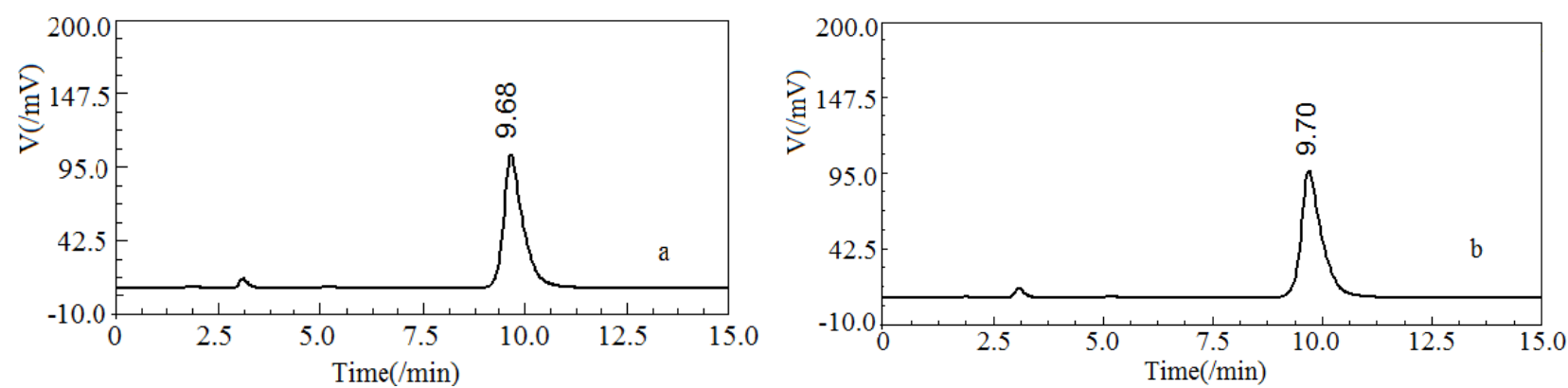

Fig. 2 The retention time of paclitaxel standard(a) and paclitaxel coated(b)

FTIR spectroscopy. All characterized balloon samples were exhaustively cleaned to remove any free polymer before FTIR was performed. Fig. 3 shows the spectrum of the balloon raw material and coated balloon removed coating. The N-H bending vibration absorption peak and methylene C-H stretching vibration absorption peak is at 3290 and $3084 \mathrm{~cm}^{-1}$, respectively. The absorption peak at $2920 \mathrm{~cm}^{-1}$ is from methylene asymmetry stretching vibration, and at $2847 \mathrm{~cm}^{-1}$ is from methylene symmetry stretching vibration. The double absorption peaks at 1652 and $1549 \mathrm{~cm}^{-1}$ are designed to the amide group. The $\left(\mathrm{CH}_{2}\right)_{\mathrm{n}}$ shows the rocking vibration absorption peak at 721 $\mathrm{cm}^{-1}$. The FTIR spectroscopy of the coated balloon is very similar with the uncoated balloon. It indicates that the structure of paclitaxel coated balloon surface hasn't been influenced.

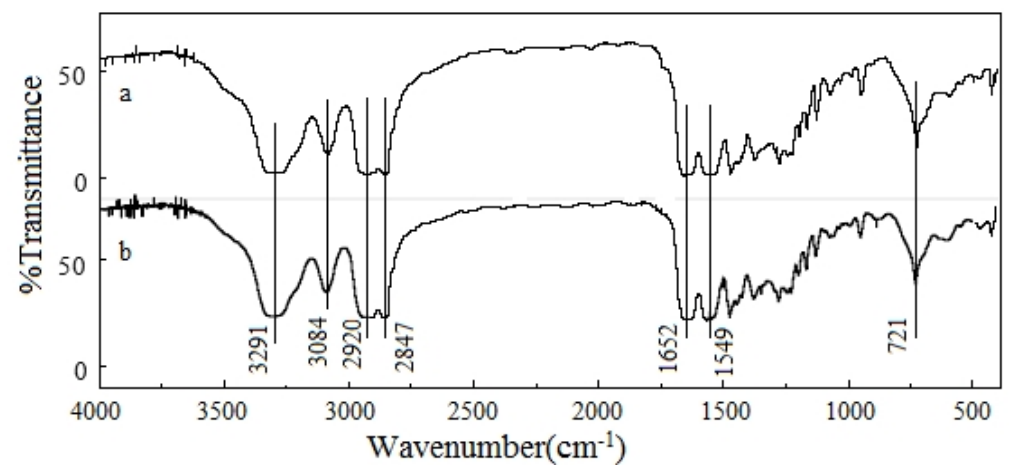

Fig. 3 The FTIR spectroscopy of uncoated balloon(a) and coated balloon(b) 


\section{Conclusions}

After coating paclitaxel drug on balloon surface, it is can been seen that other performances are no changed except the balloon diameter and balloon crossing profile were slightly increased by physical tests. Experimental results by the HPLC indicated the properties of paclitaxel on the balloon surface hasn't been changed, and the FTIR spectroscopy results showed that the structure of the balloon surface also hasn't been impacted by paclitaxel. These test results demonstrated that the balloon coated by paclitaxel still has steady and excellent performances.

\section{References}

[1] Goldman B, Blanke H, Wolinsky H, Influence of pressure on permeability of normal and diseased muscular arteries to horseradish peroxidase: a new catheter approach, Atherosclerosis. 65 (1987) 215-225.

[2] Scheller B, Speck U, Abramjuk C, et al, Paclitaxel balloon coating, a novel method for prevention and therapy of restenosis, Circulation. 110 (2004) 810-814.

[3] Scheller B, Speck U, Schmitt A, Addition of paclitaxel to contrast media prevents restenosis after coronary stent implantation, Journal of the American College of Cardiology. 42 (2003) 1415-1420.

[4] Liu Huang Jun, Ji Li Jun, Research Progress on Drug-eluting Balloon, Chinese Journal of Arteriosclerosis. 9 (2012) 857-859.

[5] Seiji H, Kazuaki M, Kazushige K, et al. Effectiveness of Paclitaxel-Eluting Balloon Catheter in Patients With Sirolimus-Eluting Stent Restenosis[J], JACC: Cardiovascular Interventions. 4 (2011) 149-154.

[6] AnikóPósa, NoemiNyolczas, RayyanHemetsberger, et al, Optimization of drug-eluting balloon use for safety and efficacy: Evaluation of the 2nd generation paclitaxel-eluting DIOR-balloon in porcine coronary arteries[J], Cathet. Cardiovasc. Intervent. 76 (2010).

[7] Ron Waksman, Rajbabu Pakala, Drug-Eluting Balloon: The Comeback Kidgo[J], Circulation: Cardiovascular Interventions. 2 (2009) 352-358.

[8] Bodo Cremers, Melanie Biedermann, Dirk Mahnkopf, et al, Comparison of two different paclitaxel-coated balloon catheters in the porcine coronary restenosis model[J], Clinical Research in Cardiology. 98 (2009) 325-330.

[9] Liu Guo zhu, Xu Li xia, Shi Xiao man, et al, Preparation of drug-eluting balloon technology to explore[J], Applied chemical industry. 44 (2014) 502-505.

[10] SchellerB, SpeckU, Romeike B, Contrastmedia as carriers for local drug delivery successful inhibition of neointimal proliferation in the porcine coronary stentmode, Eur Heart J. 24 (2003) 1462- 1467.

[11] Li Zhen, Xu Li xia, Lu Chun lan, et al, Internal Structure Changes of Nylon 12 in Balloon Forming Process, Applied Mechanics and Materials. 528 (2014) 153-161.

[12] P.W. Radke, M. Joner, A. Joost, et al, Vascular effects of paclitaxel following drug-eluting balloon angioplasty in a porcine coronary model: the importance of excipients, Eurointervention. 7 (2011) 730-737. 